NASA-CR-201887

\title{
UTILIZATION OF AN AGILITY ASSESSMENT MODULE IN ANALYSIS AND OPTIMIZATION OF PRELIMINARY FIGHTER CONFIGURATION
}

\author{
Angelen Ngan*, Daniel Biezad** \\ Aeronautical Engineering Department, Cal Poly State University \\ San Luis Obispo, CA 93407
}

\begin{abstract}
A study has been conducted to develop and to analyze a FORTRAN computer code for performing agility analysis on fighter aircraft configurations. This program is one of the modules of the NASA Ames ACSYNT (AirCraft SYNThesis) design code. The background of the agility research in the aircraft industry and a survey of a few agility metrics are discussed. The methodology, techniques, and models developed for the code are presented. The validity of the existing code was evaluated by comparing with existing flight test data. FORTRAN program was developed for a specific metric, PM (Pointing Margin), as part of the agility module. Example trade studies using the agility module along with ACSYNT were conducted using a McDonnell Douglas F/A-18 Hornet aircraft model. The sensitivity of thrust loading, wing loading, and thrust vectoring on agility criteria were investigated. The module can compare the agility potential between different configurations and has the capability to optimize agility performance in the preliminary design process. This research provides a new and useful design tool for analyzing fighter performance during air combat engagements in the preliminary design.
\end{abstract}

\footnotetext{
* Graduate Researh Assistant. Member AIAA

** Professor. Associate Fellow AIAA
}

Copyright (C) 1996 by the American Institute of Aeronautics and Astronautics, Inc. All rights reserved.

\section{Introduction}

Agility Background

Agility and flight in expanded maneuvering envelopes have been considered as ways to improve aircraft combat effectiveness, which is a combination of survivability and mission effectiveness.' Traditional aircraft performance provides a good indication of maneuverability. The most maneuverable aircraft is the one that has the highest turn rate or can pull the most g's. The increasing maneuverability of current generation fighters has pushed maximum instantaneous $\mathrm{g}$ capability to the human limit. The measure of merit has to evolve from how many g's the aircraft can pull to how quickly it can achieve this limit.

Agility is a measure of how quickly the aircraft can be maneuvered. It relates to minimizing the time required to perform some tasks or to achieve a desired aircraft state. The simplest definition of agility is the ability to move quickly in any direction or to perform a specific task. Future "superagile" vehicles will greatly expand the flight envelope with new longitudinal acceleration/deceleration capabilities, lateral and vertical direct force application, increased control authority in all axes, and increased sustained and instantaneous turning ability. The design which performed a set of maneuvers quickest would have the highest potential agility. Different sets of maneuvers will represent different versions of agility metrics. The need to define, measure, and quantify aircraft agility has been driven primarily by the inadequacy of traditional aircraft measures of merit and the emergence of advanced aircraft technologies and capabilities. ${ }^{2}$

Aircraft agility is a highly complex and integrated problem involving aerodynamics, propulsion, structures and controls. However, there are very few concrete definitions of what agility is. There are as many definitions of agility as there are researchers in this area. This has made it difficult to compare the results of one investigator with those of another. ${ }^{3}$ As of today, the absolute definition of agility is still a subject of debate. Each of the definitions of agility proposed by the government and the industry represent different quantities measuring the performance capability of an aircraft. ${ }^{4}$ The same aircraft could be less agile in one 
sense and more agile in another. Some of the proposed definitions by the government and industry in an effort to define and measure aircraft agility can be found in Reference 17.

Since the pilots, engineers and researchers now involved in agility have not yet reached a commonly accepted definition of the term, it is not surprising that the proposed agility metrics deal with many different aspects of fighter capability. The various metrics proposed to measure agility deal in units of time, velocity, angular rate, distance and combinations of time, rate and distance.

The existence of many definitions indicate a lack of standardization. There is little agreement on what agility is, even on the most fundamental level. Although agility is determined by a combination of performance and handling quality characteristics of the aircraft, it is very difficult to completely define and apply agility through our present state of knowledge of either flying qualities and/or maneuvering performance. $^{13}$ Agility is a function of both maneuverability and controllability. The most maneuverable aircraft is the one that can go from the initial state to the desired final state most quickly. Agility of the aircraft does not have hard limiting values which means the more agility, the better. The indirect bounds on the achievable agility of an aircraft are maximum structural loads, stability and controllability limitations, and retaining the desired flying qualities characteristics. $^{12}$ Some of the published agility metrics can be found in Reference 17.

\section{ACSYNT Background}

The ACSYNT (AirCraft SYNThesis) program for aircraft conceptual design was developed at NASA Ames Research Center during the 1970's to study the effects of advanced technology on aircraft synthesis. ACSYNT is a conceptual design code that is designed in a modular fashion, with each discipline of aircraft design analysis assigned to a different module or structured group of routines intended to handle that particular phase of analysis. Current ACSYNT analysis modules include Geometry, Trajectory (mission profile and performance), Aerodynamics, Propulsion, Stability and Control, Weights, Supersonic Aerodynamics, Economic, Agility and Takeoff and Landing. Using these modules, the code can analyze supersonic or subsonic transports, fighters, and bomber aircraft. ACSYNT's modular structure lends itself to optimization techniques. The optimization program COPES/CONMIN is coupled with the current version of ACSYNT. COPES (Control Program for Engineering Synthesis) / CONMIN (Constrained Minimization) gives users the ability to perform sensitivity analysis, optimization, two-variable function space analysis, and approximate optimization using ACSYNT variables and analysis methods for up to 128 constraints and/or objective functions. The ACSYNTCOPES package performs trade studies and evaluates the impact of technologies on configurations. Improvements in materials, propulsion and other technologies can be incorporated and their effect on aircraft configurations can be readily determined.

\section{Objectives of the Research}

The importance of agility is to provide a combat advantage over other aircraft. The goal for the agility study is to develop a methodology for inclusion of agility based requirements in aircraft conceptual design decisions. The design method is to provide quantitative estimates of aircraft agility characteristics and to be applied as a part of the optimization loop in future fighter aircraft design. The agility module in ACSYNT provides analysis of agility metrics and agility criteria. Implementation of technologies to improve aircraft agility are analyzed and optimized in ACSYNT while their penalty and impact on other design constraints are determined. This analysis provides some insight into the utility of agility technologies and the combat effectiveness of an aircraft configuration.

\section{Agility Metrics}

The general character of the agility module is to operate on the upper boundary of what is frequently referred to as the doghouse plot. This is a graph of turn rate versus Mach number at a specified altitude. Figure I illustrates a typical doghouse plot. The peak in the upper boundary represents the highest turn rate for any Mach number. The Mach number corresponding to the peak is usually called corner speed. The aircraft's turn rate is limited by different constraints depending on which side of corner speed it is flying. Above corner speed, the aircraft can aerodynamically generate a higher load factor than the aircraft's structure can withstand. The aircraft is said to be "load limited" with the maximum turn rate determined by the maximum designed load factor. Below corner speed, the aircraft is operating at its maximum lift coefficient and cannot aerodynamically generate the design load factor. This region is said to be "lift limited." The definition of corner speed can be said as the Mach number that produces the maximum design load factor at maximum lift coefficient. In a dogfight, pilots try to get to corner speed as quickly as possible as it provides the best turn rate. Two specific metrics are discussed because they are being developed as part of the ACSYNT agility 
module. The metrics discussed illustrate the differences of opinion on what agility is. Some analyze how efficiently aircraft use energy to achieve an objective and how quickly they can regain lost energy. Other metrics analyze the quick-action nose pointing capability of a configuration. The agility module developed is adaptable enough to accommodate several philosophies and their respective metrics.

\section{Combat Cycle Time (CCT)}

The combat cycle time metric measures the time it takes to turn through a specified heading change and then accelerate to regain the energy lost during the turn. The exact maneuver is as follows: roll into turn, pitch to specified load factor, hold turn through specified heading change, pitch back down to unity load factor, roll to wings level and accelerate back to original speed. The objective is to complete this maneuver in the least amount of time. In this maneuver the aircraft operates along the upper boundary of the doghouse plot. Figure 2 illustrates the path the aircraft follows on this plot over the course of the maneuver.

\section{Pointing Margin (PM)}

Aircraft nose pointing at the adversary in shorter time will be one of the primary capabilities required in the design of future agile fighters. Pointing the nose/weapon of the aircraft at the adversary first will be required to win, since pointing first means having the opportunity to shoot first. ${ }^{14}$ The pointing margin metric measures how fast an aircraft can point his nose at an adversary aircraft. This parameter is a function of flight condition, mach number, altitude, and heading angle of the turn. The maneuver is shown in Figure 3. The two aircraft begin at the same Mach number and nearly the same location in space but pointed in opposite directions. At the start of the metric both aircraft begin a maximum acceleration turn toward one another. The aircraft that first brings his line of sight upon the opposing aircraft's position is considered the most agile. Pointing margin for the friendly fighter is the angle between the nose of the adversary aircraft and the line of sight joining the two fighters at the instant the friendly fighter first points its nose/weapon at the adversary's aircraft. The greater this angle the longer it takes the losing aircraft to acquire the winning aircraft's position. This provides the winning aircraft a longer missile flight time and a better chance of a kill. A firstshot capability is considered a vital edge in winning aerial combat.

\section{$\underline{\text { Method }}$}

This research consists of two parts, a validation phase and a methodology enhancement phase. Validation consists of evaluating a present inventory fighter against existing maneuver data. Methodology enhancement will involve identifying new, unsupported agility metrics and adding them to the existing code framework. A currently unsupported agility metric, Pointing Margin, has been written and added to the existing code framework. The improvements made to the aerodynamics, propulsion, and mass properties modules of ACSYNT were incorporated into the existing agility metric analysis. An effort was made to enhance the existing module by implementing stability and control power derivatives which would modify the governing equations. Digital Datcom can be used currently to obtain stability and control derivatives at different angle of attacks and altitudes. Development and implementation of this module would allow the user to time step through a sequence of maneuvers to evaluate the time and positional performance of a given aircraft configuration.

\section{General Methodology}

The overall structure of the code is a timestepping routine that tracks pertinent parameters over the course of the agility maneuver. This is basically a simulation technique. Since CCT and PM were selected as archetypes for the simulation package, there exists separate subroutines dedicated to analyzing those metrics. There are two options to evaluate the other agility metrics. The user may input the desired maneuver segments into an existing agility subroutine or may create a different agility subroutine with different maneuver segments and parameters. The assumptions that were made throughout the development of the flight mechanics can be found in Reference 16.

\section{Flight Dynamics}

The agility metric maneuvers were divided into separate segments. Figure 4 illustrates the four types of maneuver segments: rolls, pitches, turns, and accelerations.

Agility metrics are categorized by time scales (transient, functional) or by the type of motion involved (lateral, pitch, axial). Functional maneuver segments deal with long-term changes (>5 seconds) in aircraft energy state, position and attitude. They quantify how well the fighter executes rapid changes in heading or rotations of the velocity vector. Emphasis is on energy lost during turns through large heading angles and the 
time required to recover kinetic energy after unloading to zero load factor. Equations of motion for the functional segments were steady-state equations for turns and rectilinear flight. Transient maneuver segments deal with short-term changes ( $1-5$ seconds) in aircraft accelerations, positions and orientation. They quantify the fighter's ability to generate controlled angular motion and to transition quickly between minimum and maximum levels of specific excess power. Equations of motion for the transient segments were standard longitudinal and lateral-directional perturbation equations.

The present module is best suited for functional type metrics because ACSYNT's stability module is not fully operational and the flight control module is not yet incorporated. Once those modules are fully operating, the transient maneuver analysis capabilities will be improved. Currently, the transient metrics may be analyzed, but the analytical models are not as robust as for the functional type segments.

\section{Equations of Motion for Functional Maneuver Segments}

The turn subroutine is designated as quasisteady since the turns are not assumed to be sustained, which makes Mach number a variable. Thus, the aircraft thrust and lift/load limit properties vary through a turn. The acceleration subroutine returns the thrust vector to the horizontal, throttles up to full power and simply accelerates the aircraft through a user specified maciı number range while maintaining straight and level flight.

\section{Equations of Motion for Transient Maneuver}

\section{Segments}

Pitch and roll subroutines maneuver the aircraft to a user designated load factor and bank angle, respectively. The pitch equations of motion were standard two degree of freedom short-period approximation equations. The roll segments were modeled with a single degree of freedom, lateral equation of motion. It uses aileron effectiveness and roll damping to construct a single degree of freedom roll schedule.

\section{Code Options and Features}

The agility operating code contains some options and features for the users to customize the maneuvers by manipulating the input parameters. These features include the angle of attack limiter, throttle control and turning speed capture, thrust vectoring, air brake, external stores release and weight/moment of inertia control.

\section{Code Verification}

For an accurate code verification, the agility module should be checked against flight test data. Therefore, validation was performed to compare agility analysis with the existing maneuver data of an inventory fighter. The only flight test maneuver data available was from one of the NASA Dryden Flight Research Facility's F/A-1 8 HARV flight tests. The flight test data contained a very comprehensive list of parameters except for the positional tracking, namely, XYZ positions. The positional comparison could not be completed in light of the lack of data. The parameters being compared are time, mach number, heading angle, roll rate, bank angle, load factor, angle of attack, and turn rate. The technique that is used for the validation is called simulation matching in which the real data is being tested in the code to see if it produces similar result.

A test was performed to ensure the code was working properly for the individual segments, such as roll, pitch, etc. This was done by testing piecewise segments. The piecewise test proved that the code provides acceptable result for each individual segment. Theoretically speaking, a complete maneuver should be performed the same way as when different segments are added together, if each piece is performed as expected. The flight test data was composed of many different random segments of maneuvers, and it was not in any easily identified classical maneuver such as Combat Cycle Time or Pointing Margin. Each segment has its own boundary conditions, therefore it was very difficult to mix and match them to create a classical maneuver. The next task was to simulate the whole maneuvers. The major problem was to decompose a continuous maneuver into the appropriate discrete segments. As expected, there is always deviation between theory and reality. The pilot may perform a roll and a pitch simultaneously instead of a discrete pitch after a discrete roll. Another problem was not knowing exactly when each maneuver began and ended. The fighter was maneuvering with a combination of different segments in a short time and data was recorded in an interval of 0.5 sec. A test run was finally generated with a maneuver that is very similar to the CCT (roll-pitchturn-pitch-roll-accel). As stated above, it was extremely difficult to identify where and each segment begins and ends. It is a matter of judgment concerning the identification of the different segments in the test data. Guidance for the decision is found by looking at the maneuver characteristics such as maintaining a constant AOA for a turn, constant roll rate and bank angle for a pitch, or constant load factor for a roll. The predicted maneuver is obviously not what the fighter was actually doing, but it was believed to be close enough for our 
purposes. It is understood that a continuous reality can not be simulated completely by discrete simulation. With the above information, the appropriate parameters were supplied and initialized in the code according to the test data. It was found that controlling these boundary conditions was critical, since the original code initialized those parameters to be zeros, changes had to be made in the appropriate subroutine. Other than these necessary inputs, the code was not changed in anyway.

While results were very good, there are several factors that introduce errors in this validation. Any difference between the simulated maneuver and the actual maneuver is going to cause the error in the analysis. One source of error is a discontinuity between segment boundary conditions. Figure 5 shows mach number vs. time for a typical maneuver. As seen on this graph, the matching is quite good. The average percentage error between the actual and the ACSYNT curve is $0.21 \%$. The discontinuities in the graph can be seen more clearly in Figure 6. This figure shows actual, ACSYNT, and ACSYNT-Modified curves. The discontinuity is located at the transition from one segment to another. The ACSYNT-Modified curve is generated by assuming that the curve is continuous instead of discrete. It shows how the curve should be without the discontinuity between each segment. The difference between the ACSYNT and the ACSYNTModified results due to the fact that the boundary conditions between segments are not forced to be the same in the code. If the boundary conditions of the beginning of a segment are the same as the end of the previous segment, then a piecewise continuous analysis can be obtained easily. When there is only one boundary condition, the analysis is continuous by definition. Another source of error has to do with simulation vs. reality. As shown in Figure 7, the curves clearly distinguish the behavior of a real and a simulated maneuvers. For a real maneuver, the flight is very smooth with a gradual increase in the load factor. Conversely, the simulated flight jumps to the designated g's for each segment. This would certainly contribute errors into the validation. Comparisons between heading angle, bank angle, load factor, turn rate, and angle of attack with time and mach number were made. For all of these comparisons, the percentage errors are shown in Table 1. Again, the discontinuity in the curve is caused by not forcing boundary conditions between maneuver segments to be the same in the discrete analysis. As can be seen in Table 1, the percentage error is acceptable for this kind of analysis. Thus it can be concluded that this validation analysis is satisfactory and the existing computer code is valid.

\section{Trade Studies}

\section{Effect of Thrust Loading and Wing Loading}

Thrust Loading (T/W) and Wing Loading (W/S) are the two most important parameters affecting aircraft performance. An aircraft with a higher $T / W$ will accelerate more quickly, climb more rapidly, reach a higher maximum speed, and sustain higher turn rates. However, the larger engines will consume more fuel throughout the mission, which will drive up the aircraft's takeoff gross weight to perform the design mission. W/S affects stall speed, climb rate, takeoff and landing distances, and turn performance. Wing loading determines the design lift coefficient, and impacts drag through its effect upon wetted area and wing span. Wing loading has a strong effect upon sized aircraft takeoff gross weight. If the wing loading is reduced, the wing is larger. This may improve performance, but the additional drag and empty weight due to the larger wing will increase takeoff gross weight needed to perform the same mission.

The studies performed are intended to illustrate how the agility module may be used to ascertain and optimize an aircraft configuration's agility potential. The two parameters were chosen because they are fundamental in classical energy maneuverability analysis as discussed earlier. The new agility metric analysis shows aircraft that appear to have similar energy maneuverability performance levels can have quite different levels of agility. The baseline aircraft used for these studies was the McDonnell Douglas F/A-18 Hornet. The weights, external dimensions and installed thrust were matched to obtain a representative fighter model. The maneuver used was a $7 \mathrm{~g}$ turn through 180 degrees at an altitude of 15,000 feet beginning at straight level flight and Mach 0.9. A pointing margin (roll-pitch-turn) maneuver was performed for the test runs. The effects on $T / W$ and W/S on PM are discussed.

\section{Effect of Thrust Loading on Pointing Margin}

The baseline fighter along with four other configurations were flown through the same maneuver. These configurations were altered only in the available level of thrust specified as a percentage of the baseline configuration's available thrust $(80 \%, 90 \%, 110 \%$, $120 \%$ ).

Figure 8 illustrates the time differences for each segment of the pointing margin maneuver for all five configurations. The maneuver times steadily increased with increased available thrust and the lowest thrust aircraft performed the maneuver in the least amount of time which also implies that the lower thrust aircraft completed the turn segment slightly quicker 
than the higher thrust aircraft.

Turning speed determines an aircraft's highest turn rate. It is understandable why the lower thrust aircraft completed their turns sooner. Their higher decelerations placed them in speed regimes with higher turn rate than the greater thrust aircraft and thus were able to achieve superior turns. If the starting velocity were below the turning speed, the higher thrust aircraft would be better able to accelerate to and maintain the turning speed. It is situations like this that make the development of agility criteria so difficult. The configuration can be entirely dependent on the specific situation. Figure 9 illustrates the turn profile in the horizontal plane of the maneuver. The lower thrust configurations turn tighter and possess a positional advantage over the course of the turn segment.

Figure 10 showed pointing margin vs. thrust loading. A better pointing margin can be obtained for a lower thrust loading which is consistent with the turning speed effect that was discussed. The aircraft that reaches the turning speed and completes the turn sooner can always obtain a better positional advantage.

The impact of thrust loading is entirely dependent on what is considered most important. For PM type of maneuver, a lower thrust aircraft would be a better choice because lower thrust configurations possessed a positional advantage up to the end of the turn segment. The conclusion of this study is there is a tradeoff of what type of performance is most crucial and what are its costs.

\section{Effect of Wing Loading on Pointing Margin}

The baseline fighter along with four other configurations were flown through the same maneuver. These configurations were altered only in the wing loading and all other input parameters were held constant. The selected wing loadings were 82.6, 87.6, 97.6, and $102.6 \mathrm{psf}$ with a baseline W/S of $92.6 \mathrm{psf}$.

Figure 11 illustrates the time differences for each segment of the pointing margin maneuver for all five configurations. The total time to complete the maneuver was very similar for all configurations, but there was a difference in the times for each maneuver segment. The higher loaded aircraft completed the turn segment slightly faster than the less loaded configurations. This is because a higher loaded aircraft produces higher lift coefficients, thus increases induced drag and results in greater deceleration and velocity deficit. Similar to the thrust loading results, the quicker approach to turning speed provided higher turn rates and resulted in a shorter time for a turn. Figure 12 plots the turn profile in the horizontal plane of the maneuver. This graph shows the higher loaded aircraft has a turn advantage both in time and in space. The points discussed above are also well illustrated in Figure 13. It shows that a better pointing position can be obtained with a higher wing loading which correspond to the fact that a higher wing loading has a turn advantage.

Again, it was illustrated that the results of this study were highly dependent on the particular type of maneuver. If we were looking at some other maneuvers, a higher W/S may not be desired. This shows the difficulty in developing robust agility criteria that provide the best overall performance for a variety of situations and tasks.

\section{Effect of Thrust Vectoring on Pointing Margin}

Vectored thrust offers improved turn performance for future fighters, and is used in the VSTOL fighter to maximize turn-rate. The direction that the thrust should be vectored to depends upon whether instantaneous or sustained turn-rate is to be maximized. In a level turn with vectored thrust, the load factor times the weight must equal the lift plus the contribution of the vectored thrust. For a lift limited turn, thrust vector for maximum instantaneous turn-rate should be perpendicular to the flight direction while the thrust vector for maximum sustained turn rate should be aligned with the flight direction. Thrust vectoring capability of the agility module does not include pitch control thrust-vectoring. Instead it includes the ability to rotate the thrust vector out of the fuselage axis yet remain centered at the aircraft's center of gravity. This is intended to model the in-flight direct lift capability of aircraft.

A vectored thrust study was conducted to determine the effects of this capability on horizontal maneuver. The metric used for the study was Pointing Margin. As mentioned previously, the figure of merit is the angle between the two aircrafts' lines of sight just as the inferior aircraft is captured. The F-18 fighter is considered as a point mass and is confined to the horizontal plane. Thrust vector capability is included in the F-18 fighter. The maneuver used was a $7 \mathrm{~g}$ turn through 180 degrees at an altitude of 15,000 feet. In actual combat, nozzle position would most likely be fully variable throughout maneuvers. For this study however, it was restricted to three possible positions during two segments of the maneuvers. A nozzle position angle of zero degrees indicated thrust along the longitudinal axis of the aircraft. Ninety degrees represented thrust vectored normal to the longitudinal axis, while forty-five degrees indicated the thrust was vectorally split between these two directions. The scheduling of the thrust vector angles was separated by corner speed. The nozzle position was fixed at one of the three positions while the aircraft was above corner speed and then switched to another as it decelerated below corner speed. This scheduling and the three 
nozzle positions provided a good range of possible vectoring tactics for evaluation. For all figure labels, two numbers separated by an underscore indicates the VT nozzle position before and after reaching corner speed, respectively.

The effects of vectored thrust (VT) are apparent from PM. The influence of VT scheduling on PM is indicated in Figure 14 and the zoom-in view in Figure 15. It is clear that a better pointing margin can be obtain by having thrust vectoring capability. The VT effect was able to capture two more degrees of pointing advantage which provides the winning aircraft a longer missile flight time and a better chance of a kill. The greater this angle the longer it takes the losing aircraft to acquire the winning aircraft's position. As would be expected, VT is shown to decelerate much more quickly. The loss of axial thrust to combat drag results in velocity changes orders of magnitude larger than if no VT had been implemented. Figure 16 is the turn plot for various VT tactics. In each case, the VT turns show a reduction in turn radius and a corresponding positional advantage over the baseline.

The overall conclusion is that VT tactics have an apparent advantage in PM analysis and it is good from a positional aspect. Positional advantage (reduced turn radius) is particularly useful in nose-to-nose turns while time advantage (turn rate) is most useful for noseto-tail engagements.

Aircraft Optimization with Agility Parameter as One Constraint

Agility module can be used in configuration optimization. This capability is the real power of ACSYNT and it is the optimization studies that will be used to determine the impact of agility technologies and constraints on the overall aircraft configuration.

The basic optimization method used by COPES in conjunction with ACSYNT consists of an objective function, design variables and constraint variables. The objective function is the parameter being optimized and can be either maximized or minimized. Design variables are the parameters whose values are varied to provide a design space. These design variables are given upper and lower bounds. The constraint variables are parameters that further limit the design space. Typical constraints in ACSYNT are overall aircraft density, mission range, or sustained turn requirement at altitude. Only the design variable space that satisfies all constraints can provide possible solutions. The optimizer evaluates aircraft configurations over this design space and attempts to find the design point that produces the best value of the objective function.

In this case study, the objective function was gross takeoff weight. For the pointing margin maneuver with an F18 and an F20, the F18 was able to gain a positional advantage and to obtain a pointing margin of $37.15^{\circ}$. The objective for this optimization test run was to minimize the takeoff weight for the F18. Note that only the F18 is being optimized, and not the F20. The constraint for this optimization was to complete the same maneuver with a minimum pointing margin of $37.15^{\circ}$. Figure 17 illustrates the positional plot for the pointing margin maneuver for an F20 and an F18 before and after the optimization. The design variables were the wing area and the engine size. Table 2 lists the design variables bounds, the constraint variable value, and the pertinent parameters of the starting configuration and the optimized configuration. The graphical representation is illustrated in Figure 18.

The tradeoff is wing loading versus thrust loading. A decrease in wing loading allows a decrease in thrust loading and vice versa. A larger wing and a larger engine both add weight to the vehicle. Some combination of wing and engine size will satisfy the agility constraint and provide the overall lowest takeoff weight. It can be seen on Figure 18 that the trends drive the wing to as small a value as possible. This results in only a moderate increase in engine size. It is shown that the agility criterion is much more sensitive to engine size than wing loading. In real life, any functional aircraft configuration would have many more constraints such as takeoff and landing performance. Those constraints would require a much more reasonable wing size. Nevertheless, this example demonstrates the capability of ACSYNT to use agility constraints in configuration optimization.

\section{Conclusions and Recommendations}

FORTRAN programs were developed and validated for two specific metrics, CCT (Combat Cycle Time) and PM (Pointing Margin), as part of the agility module in ACSYNT design code. This module is an effective design tool in analyzing an aircraft configuration's agility potential. The integrity of the code was proved by comparing with existing flight test data. Example trade studies or the effect of thrust loading, wing loading, and thrust vectoring illustrate how the module can be used to perform trade studies on parameters important to agility metrics that are based on flight test maneuvers. The module is capable of providing constraints for ACSYNT's optimization analysis. Once agility criteria has been developed the module can be used to optimize an aircraft configuration taking into account agility requirements as well as mission requirements.

The present module is best suited for functional type metrics, particularly combat cycle time, 
pointing margin, and dynamic speed turn. Although the transient metrics may be analyzed and the architecture is well suited for transient maneuvers, the analytical models are not as robust as for the functional type segments. Once ACSYNT is capable of generating stability derivatives and the flight control module is incorporated, the transient maneuver analysis capabilities will be improved.

The validation result has proved that the code is valid for agility analysis. However, the error can be reduced by forcing the boundary conditions between maneuver segments to be the same in the discrete analysis. This can be fixed in the code easily.

The agility module's architecture is an important characteristic for future improvements. Since industry and government have not yet settled on a single definition of agility, an accepted group of metrics, nor quantifiable requirements, the adaptable architecture will allow future metrics and requirements to be incorporated with the least amount of work. The simulation's time-stepping technique of analysis and list of maneuver segments should provide the necessary adaptability. Combat Cycle Time and Pointing Margin are the two dedicated subroutines. Future work effort should involve development of subroutines dedicated to performing other agility metrics. Many metrics are appropriate for inclusion in the agility module.

The goal for this agility study is to develop a methodology for inclusion of agility based requirements in aircraft conceptual design decisions. This is accomplished by using the agility module to provide quantitative estimates of aircraft agility characteristics and to apply as a part of the optimization loop in future fighter aircraft design.

\section{Acknowledgments}

The authors are grateful Mr. Andrew Hahn, Mr. Paul Gelhausen, Mr. George Kidwell of NASA Ames, and Mr. A] Bowers of NASA Dryden for their guidance and support throughout this study. This research was funded by NASA Grant NCC2-834.

\section{References}

'Cord, T.J., "A Standard Evaluation Maneuver Set for Agility and the Extended Flight Envelope - an Extension to HQDT," AIAA Paper 89-3357, Proceeding AIAA Guidance, Navigation and Control Conference, Boston, MA, August 1989.

${ }^{2}$ Butts, Stuart, and Lawless, "Flight Testing for Aircraft Agility," AIAA Paper 90-1308, AIAA/SFTE/DGLR/SETP Fifth Biannual Flight Test Conference, Ontario, California, May 1990.
${ }^{3}$ Mazza, C.J., "Agility: A Rational Development of Fundamental Metrics and their Relationship to Flying Qualities," AGARD Conference Proceedings No. 508, Flying Qualities, Paper No. 27, October 1990.

${ }^{4}$ Bitten, R., "Qualitative and Quantitative Comparisons of Government and Industry Agility Metrics," AIAA Paper 89-3389. AIAA Atmospheric Flight Mechanics Conference, Boston, Massachusetts, August 1989

${ }^{5}$ Meeting Notes, AFFDL Specialists Meeting on Agility, July 1986

${ }^{6}$ AFFTC Workshop on Agility, March 1988

${ }^{7}$ Northrop F-20 Marketing Brochure.

${ }^{8}$ McAtee, T. P., "Agility - Its Nature and Need in the 1990 's," Presented at the Proceedings of the $31 \mathrm{st}$ Symposium of the Society of Experimental Test Pilots, Beverly Hills, Ca, September 1987.

'Herbst, W.B., "Agility," Briefing Presented at the Workshop on Agility Metrics Held at the Air Force Flight Test Center, Edwards AFB, Ca, 8-10 March 1988.

${ }^{10}$ USAF Test Pilot School Class 87B Report on "Ultimate Agility."

"Skow, A. M., et. al, "Transient Agility Enhancements for Tactical Aircraft," Eidetics International TR89-001, Prepared Under USAF Contracts F33615-85-C-0120 and F33657-87-C-2045 for ASD/XRM, January 1989.

12 Kalviste, J., "Measures of Merit for Aircraft Dynamic Maneuvering," SAE Paper 901005, SAE Aerospace Atlantic, Dayton, Ohio, April 1990.

${ }^{13}$ Stellar, M., and Schrage, D., "An Investigation of Aircraft Maneuverability and Agility," AIAA Paper 904888 .

14 Tamrat, B.F., "Fighter Agility Assessment Concepts and Their Implications on Future Agile Fighter Design," AlAA Paper 88-4400, AIAA/AHS/ASEE Aircraft Systems, Design and Operations Meeting, Sept. 1988.

${ }^{15}$ Spearman, M. L., "Some Fighter Aircraft Trends," AIAA Paper 84-2503, AIAA/AHS/ASEE Aircraft Systems, Design and Operations Meeting, Oct. 1984.

${ }^{16}$ Bauer, B., "Analysis and Optimization of Preliminary Aircraft Configurations in Relationship to Emerging Agility Metrics," Master of Science Thesis, Cal Poly State University, Ca, December 1993.

17 A. Ngan, B. Bauer, D. Biezad, and A. Hahn, "Development of an Agility Assessment Module for Preliminary Fighter Design," AIAA Paper 96-0088, 34th Aerospace Sciences Meeting \& Exhibit, Reno, NV, January 1996. 


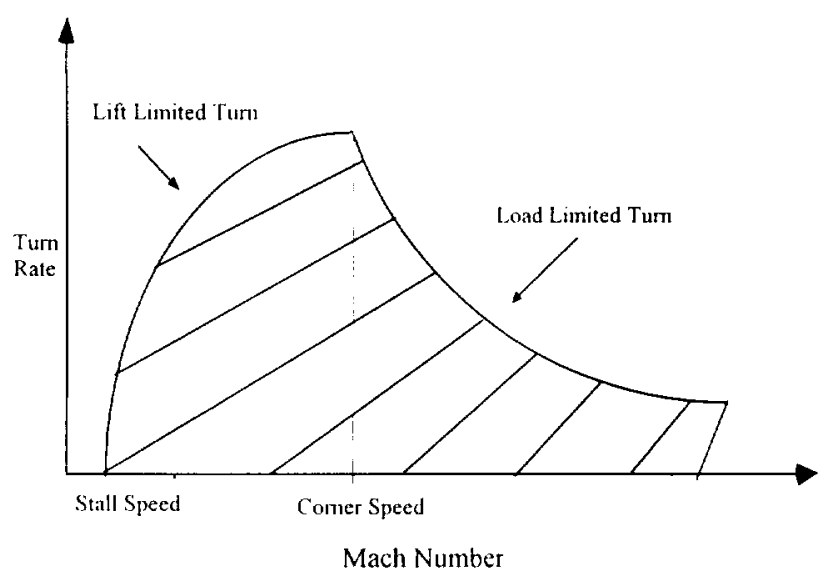

Figure 1 Illustration of the Doghouse Plot

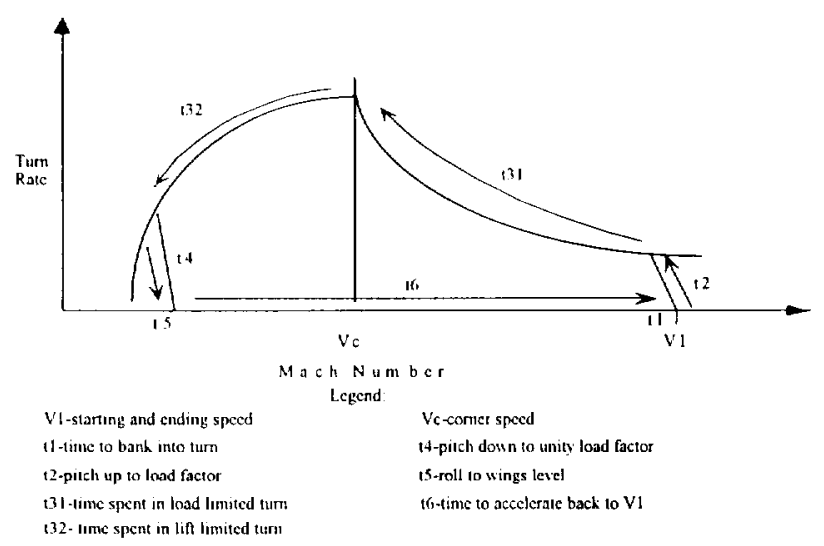

Figure 2 Combat Cycle Time Maneuver Circuit

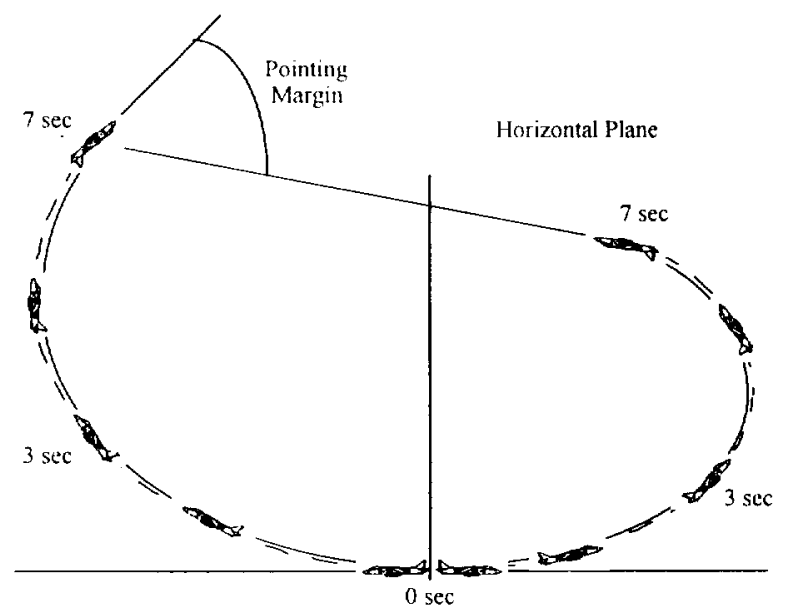

Figure 3 Pointing Margin Agility Metric

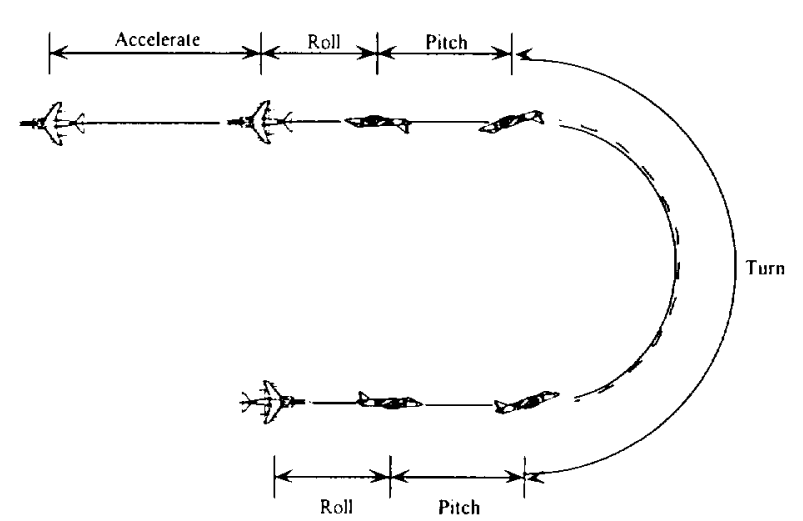

Figure 4 Breakup of Metric Maneuvers into Maneuver Segments

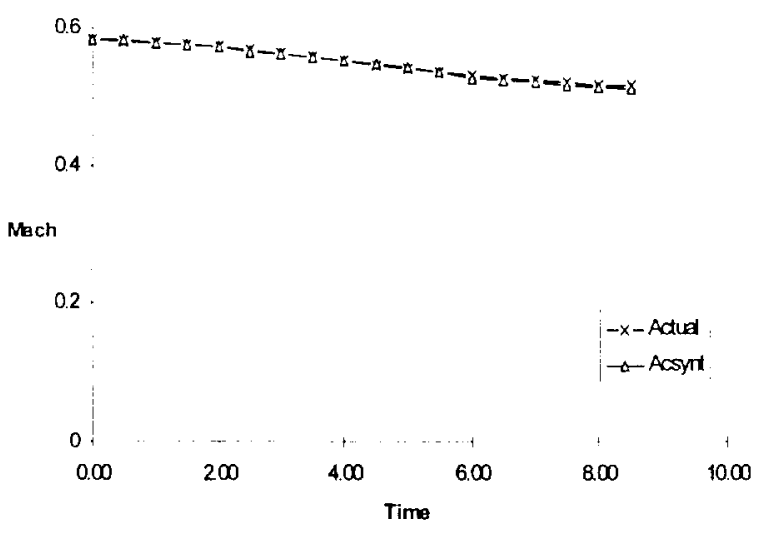

Figure 5 Comparison of Simulated and Actual Maneuvers - Mach vs. Time

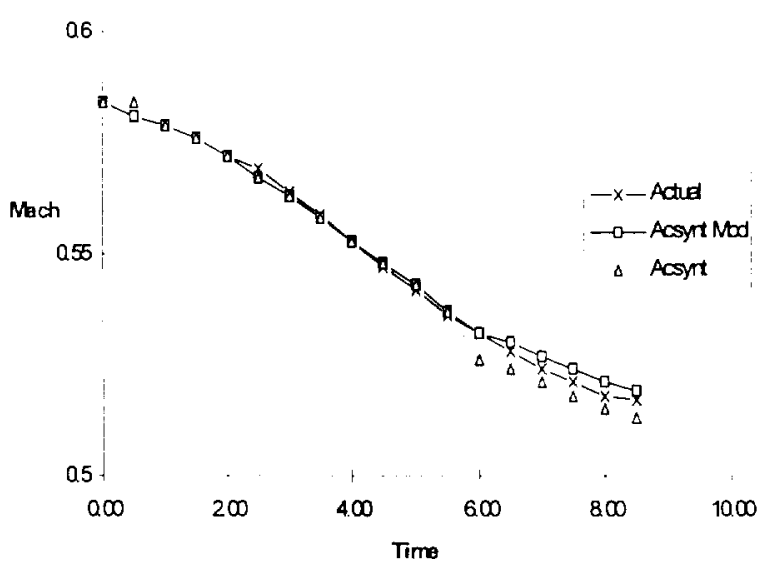

Figure 6 Comparison of Simulated and Actual Maneuvers with Modification - Mach vs. Time 


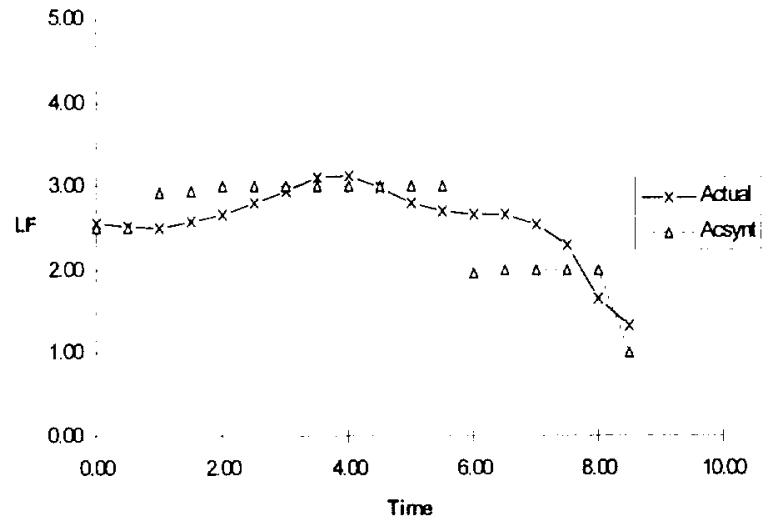

Figure 7 Comparison of Simulated and Actual Maneuvers - Load Factor vs. Time

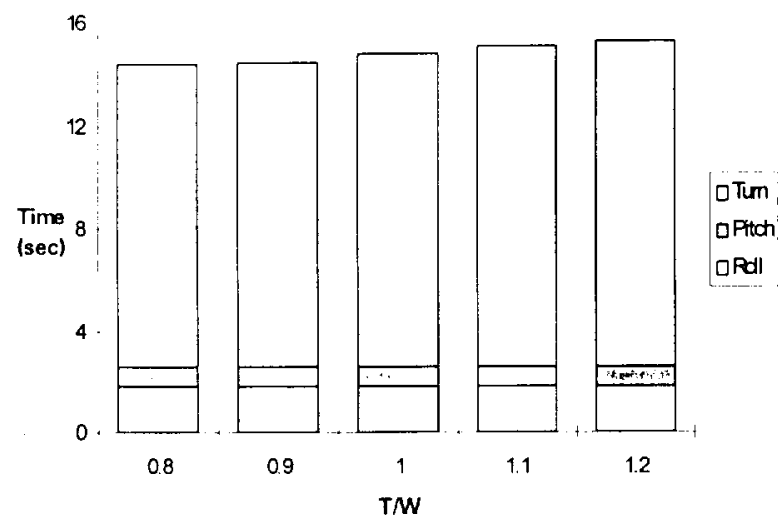

Figure 8 Pointing Margin Total Maneuver Time for Different Thrust Loadings

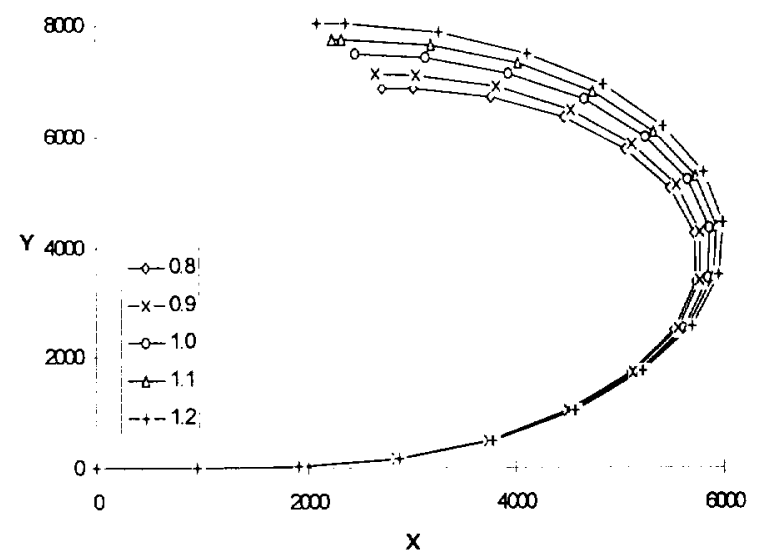

Figure 9 Horizontal Plane Turn Diagrams for Different Thrust Loadings

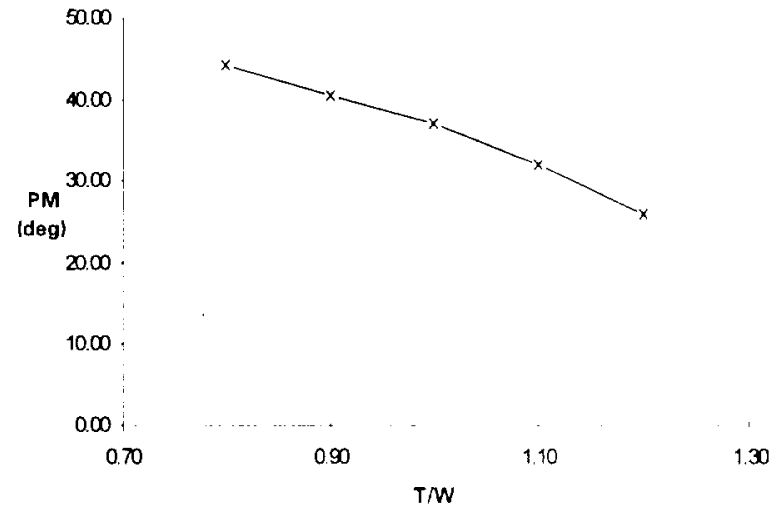

Figure 10 Pointing Margin vs. Thrust Loadings

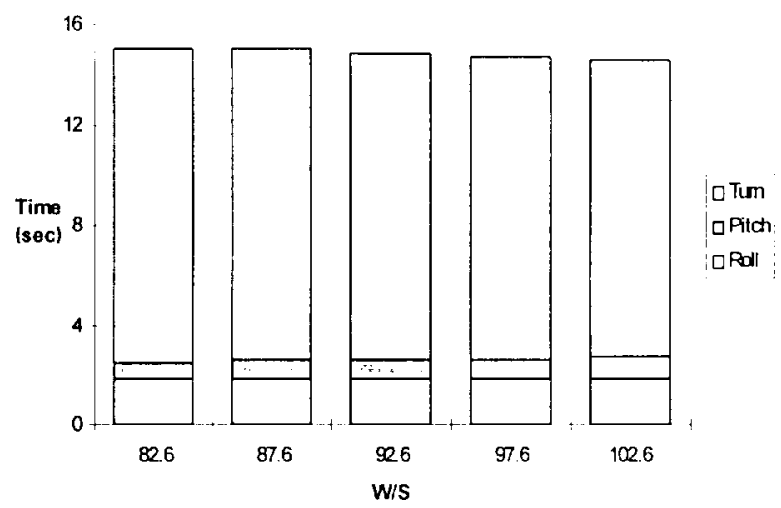

Figure 11 Pointing Margin Total Maneuver Time for Different Wing Loadings

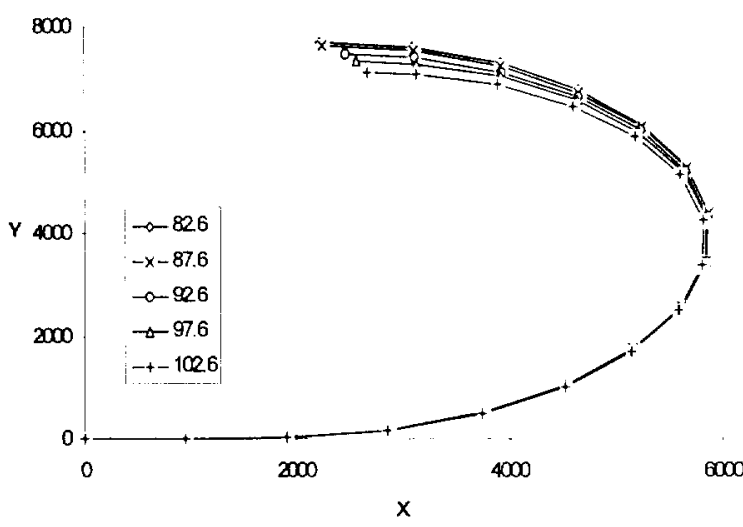

Figure 12 Horizontal Plane Turn Diagrams for Different Wing Loadings 


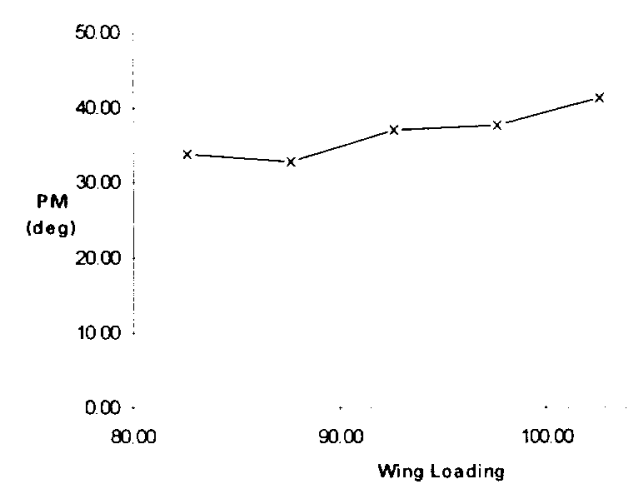

Figure 13 Pointing Margin vs. Wing Loadings

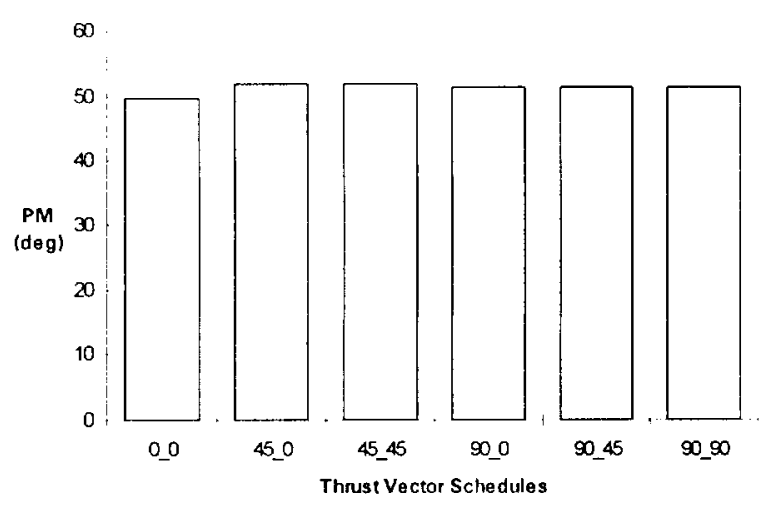

Figure 14 Effect of Thrust Vectoring on Pointing Margin

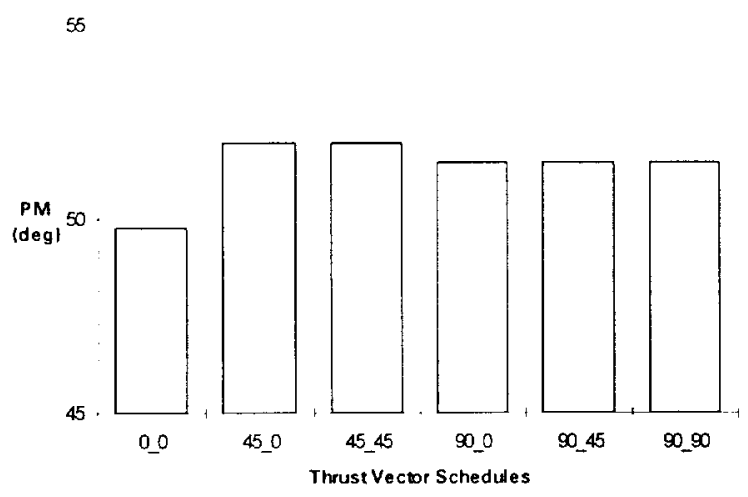

Figure 15 Effect of Thrust Vectoring on Pointing Margin -- Zoom-in View

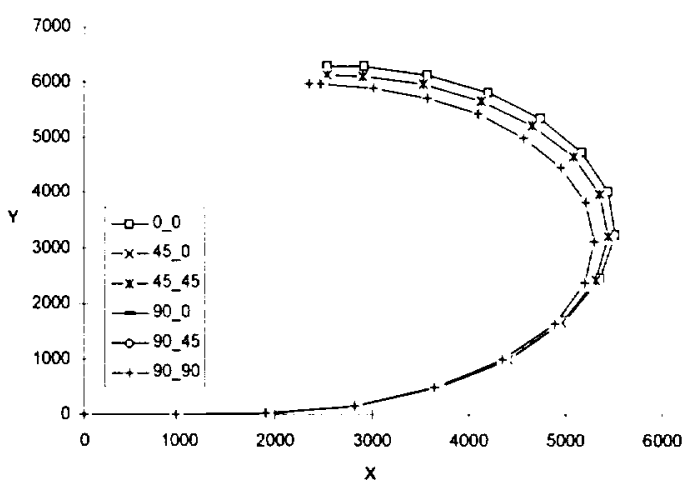

Figure 16 Horizontal Plane Turn Diagrams for Different Thrust Vector Schedule

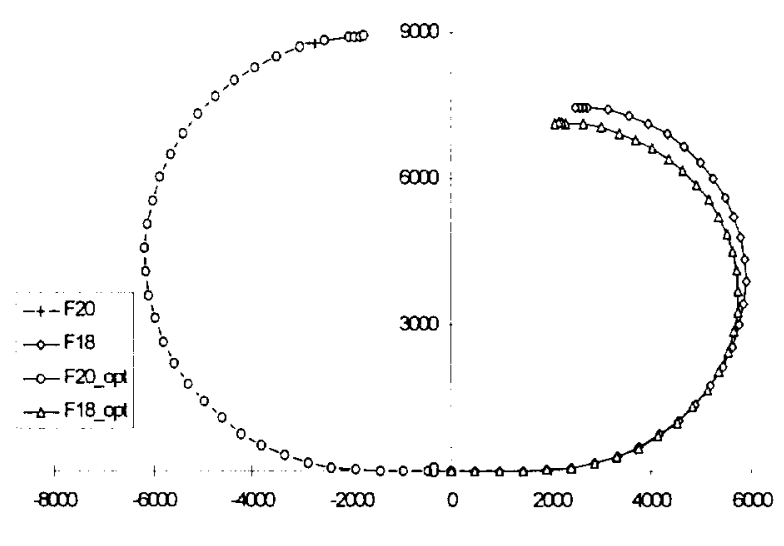

Figure 17 Pointing Margin Maneuvers for F1 8 and F20 with and without Optimization

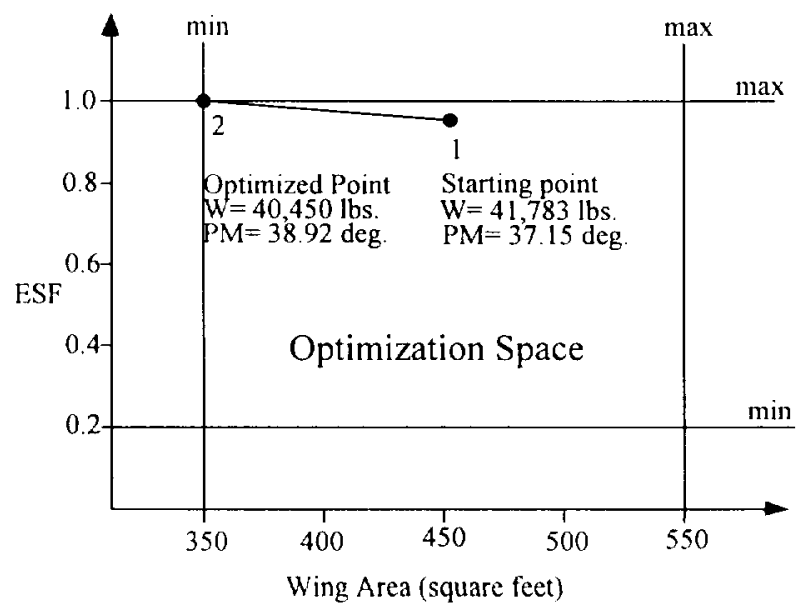

Figure 18 Optimization Path for Minimization of Aircraft Takeoff Weight 


\begin{tabular}{|l|c|}
\hline & \% error \\
\hline Mach Number & $0.21 \%$ \\
\hline Heading Angle & $0.58 \%$ \\
\hline Bank Angle & $20.70 \%$ \\
\hline Load Factor & $9.80 \%$ \\
\hline Turn Rate & $13.83 \%$ \\
\hline Angle of Attack & $17.44 \%$ \\
\hline
\end{tabular}

Table 1 Percentage Error Between Simulated and Actual Maneuvers for the Agility Code Validation

\section{Design and Constraint Variable Boundaries}

\begin{tabular}{|l|c|c|}
\hline Design Variable & $\frac{\text { Lower Bound }}{150}$ & Upper Bound \\
Wing Area $\left(\mathrm{ft}^{2}\right)$ & 0.2 & 1.0 \\
Engine Scale Factor & & \\
& $\frac{\text { Lower Bound }}{37.15}$ & $\frac{\text { Upper Bound }}{40.00}$ \\
\hline Constraint Variable & Pointing Margin (deg) & $\frac{15}{2}$ \\
\hline
\end{tabular}

Optimization Results

\begin{tabular}{|l|c|c|}
\hline Configuration & $\frac{\text { Original }}{37.15}$ & Optimized \\
Pointing Margin $(\mathrm{deg})$ & 48.92 \\
Wing Area $\left(\mathrm{ft}^{2}\right)$ & 1.1 & 350 \\
Engine Scale Factor & 1.0 & 0.937 \\
Takeoff Weight (lbs) & 41,783 & 40,450 \\
\hline
\end{tabular}

Table 2 Design Space Boundaries and Final Results for Pointing Margin Optimization 